\title{
Bi-dimensional plume generated by the convective dissolution of an extended source of $\mathrm{CO}_{2}$
}

\author{
Niloy De $\odot,{ }^{1}$ Patrice Meunier, ${ }^{2}$ Yves Méheust $\odot,{ }^{3}$ and François Nadal ${ }^{1}$ \\ ${ }^{1}$ Wolfson School of Mechanical and Electrical Engineering, \\ Loughborough University, LE11 3TU Loughborough, United Kingdom \\ ${ }^{2}$ IRPHE, CNRS, Aix-Marseille University, France \\ ${ }^{3}$ University of Rennes, CNRS, Géosciences Rennes, UMR 6118, 35000 Rennes, France
}

(Received 3 March 2021; accepted 19 May 2021; published 21 June 2021)

\begin{abstract}
The dynamics of a bi-dimensional solutal plume generated by the convective dissolution of an extended source of carbon dioxide $\left(\mathrm{CO}_{2}\right)$ is studied from both experimental and numerical standpoints. In the experiments, the $\mathrm{CO}_{2}$ is injected into a 1-mm-thick water filled Hele-Shaw cell at pressures ranging between 1 and 5 bars. The plume is visualized using a $p \mathrm{H}$-sensitive dye, and the velocity field is measured by means of standard particle image velocimetry (PIV). Concentration and velocity fields are scrutinized over one order of magnitude in the Rayleigh number (Ra), and for two different values of the Darcy number (Da). In order to extend the explored range of these dimensionless numbers, experiments are complemented by a comprehenvive set of numerical simulations. At low Darcy numbers $(\mathrm{Da}<1)$, the morphology of the numerical plume is fairly close to that of the analytical solution previously derived by Wooding [J. Fluid Mech. 15, 527 (1963)] for the case of a line source in a porous medium. At larger Darcy numbers $(\mathrm{Da}>1)$, the numerical solution departs on several aspects from this solution and exhibits closer similarity to the analytical solution derived by Spalding and Cruddace [Int. J. Heat Mass Transfer. 3, 55 (1961)] for the case of a line source in a viscous fluid. As the introduction of an additional length scale (the lateral size of the source) in the problem allows for the definition of a length-based Rayleigh number Ra, the respective amplitudes of the plume velocity $w$, plume width $\sigma$, and dimensionless total injection rate (i.e., the Nusselt number $\mathrm{Nu}$ ) can be explicitly obtained as functions of $\mathrm{Ra}$ and $\mathrm{Da}$. The scaling laws are reasonably close to those obtained from simple mass conservation considerations: (i) $w \sim \mathrm{Ra}, \sigma \sim \mathrm{Ra}^{-1 / 2}$, and $\mathrm{Nu} \sim \mathrm{Ra}^{1 / 2}$ for $\mathrm{Ra} \mathrm{Da}<1$, and (ii) $w \sim(\mathrm{Ra} / \mathrm{Da})^{1 / 2}, \sigma \sim(\mathrm{Ra} / \mathrm{Da})^{-1 / 4}$, and $\mathrm{Nu} \sim$ $(\mathrm{Ra} / \mathrm{Da})^{1 / 4}$ for $\mathrm{Ra} \mathrm{Da}>1$. Although the concentration field measurements are marred by a large uncertainty due to the logarithmic nature of the $p \mathrm{H}$-concentration relationship, the experimental data obtained from the PIV measurements show good agreement with the numerical results.
\end{abstract}

DOI: 10.1103/PhysRevFluids.6.063503

\section{INTRODUCTION}

In a world grappling with global warming, the reduction of greenhouse gas emission, such as carbon dioxide $\left(\mathrm{CO}_{2}\right)$ and methane $\left(\mathrm{CH}_{4}\right)$ is of utmost concern. In this context, carbon capture and storage (CCS) has been gaining increasing attention as a promising midterm solution to limit $\mathrm{CO}_{2}$ emissions-see Refs. [1,2] and articles referenced therein as well as Ref. [3] and related articles in the press. Even if proof-of-concept small-scale CCS sites have now proved the storage process to be efficient and reliable (e.g., Ref. [4]), assessing the storage efficiency (maximum injection rate of $\mathrm{CO}_{2}$ in the aquifer), storage capacity (maximum quantity of carbon dioxide storable 
within the aquifer) and timescales necessary for achieving perennial storage still remain a crucial challenge, and much is still to be understood regarding the physical processes at play during storage operations. Among these, the convective dissolution of supercritical $\mathrm{CO}_{2}$ into the brine saturating deep aquifers is the fundamental ingredient in the so-called solubility trapping of $\mathrm{CO}_{2}$, and much effort has been devoted to studying the gravitational fingering instability of the $\mathrm{CO}_{2}$-brine mixture and the resulting convection in different practical configurations [5]. On the laboratory scale, most experimental studies have been performed in simple vertical liquid-filled Hele-Shaw cells, using $\mathrm{CO}_{2}$ in the gas phase or analog solutes-see, e.g., Refs. [6-9] for practical implementation. Upon injection, a diffusive solute $\mathrm{CO}_{2}$-enriched liquid layer builds up at the top of the liquid phase, which is less dense than the said layer. This unstable configuration first destabilizes 3 . with a wavelength which depends on the Rayleigh number (Ra) based on the aquifer's thickness. The onset stage, subsequently, evolves towards a dynamical (nonlinear) regime where developed plumes are constantly merging with each other, whereas new plumes emerge from the free interface, thus, filling the space left vacant [10], and liquid less enriched in $\mathrm{CO}_{2}$ raises from the bottom to the upper boundaries of the liquid phase, thus, enabling continuous dissolution of the supercritical $\mathrm{CO}_{2}$ from above. A substantial effort has been made towards understanding the instability onset [11-13]. The total $\mathrm{CO}_{2}$-dissolution rate in the nonlinear regime has also been investigated by many authors both experimentally and numerically, see, e.g., Refs. $[7,13,14]$. However, hydrodynamic flow in the nonlinear regime has not been characterized in detail yet, possibly due to the extreme complexity of the observed dynamics.

A somewhat simplistic approach of the nonlinear regime consists of ignoring its unsteady nature and considering the flow as a juxtaposition of quasisteady plumes generated from extended sources of dimension $L_{s}=L /\langle N\rangle$, where $L$ is the width of the cell and $\langle N\rangle$ is the average number of plumes along the transverse dimension. As a first step towards a better understanding of the nonlinear dynamics of the plume assembly, we, thus, propose here a combined experimental and numerical study of a developed single bi-dimensional plume generated by $\mathrm{CO}_{2}$ dissolution from an extended source of lateral extension $L_{s}$ - the latter being smaller than the natural wavelength observed at the onset of the gravitational instability for a fully open Hele-Shaw cell, such as those considered in Refs. [8,9].

Several authors have derived analytical solutions for laminar plumes. In three dimensions, exact solutions were given for axisymmetric plumes generated from a point source [15-19] and from an extended source [20-22]. In two dimensions, Wooding [23] proposed a solution for bi-dimensional plumes generated from a line source in a porous medium, i.e., valid in the low Darcy (Da) number limit. Spalding and Crudacce [24] derived a solution for a plume generated by a line source in a viscous fluid, i.e., relevant to the case of large Darcy numbers. Spalding and Crudacce [24] solved the problem for large Schmidt numbers (Sc), and Fuji [16] solved the case of a Schmidt number equal to 2. However, here, we have focused on the case of an extended source.

In the case of a point source, the only relevant parameter is the Nusselt number ( $\mathrm{Nu})$ (dimensionless dissolution rate) since there is no length scale in the problem. Conversely, in the case of an extended source, its finite transverse extension $\left(L_{s}\right)$ allows for the definition of a length-based Rayleigh number and the subsequent derivation of the Nusselt number as an explicit function of the physically relevant input dimensionless parameters ( $\mathrm{Ra}$ and $\mathrm{Da}$ numbers).

The objectives of the study are to check whether the theoretical solutions known for point sources are valid in the case of an extended source and to determine the flux of the equivalent point source. The article is structured as follows. The experimental setup and measurement techniques are presented in Sec. II together with the governing equations and the numerical methods. Section III is dedicated to a general description of the experimental profiles observed in the range of Rayleigh and Darcy numbers explored experimentally. Section IV addresses the numerical investigation. The low Darcy number regime is first considered in Sec. IV A. A set of numerical results obtained at large Darcy/large Schmidt numbers (Brinkman regime) are presented in Sec. IV B. General scaling laws making the link between low Darcy and Brinkman regimes are presented in Sec. IV C. The experimental results are finally displayed together with the numerical data as functions of the key 


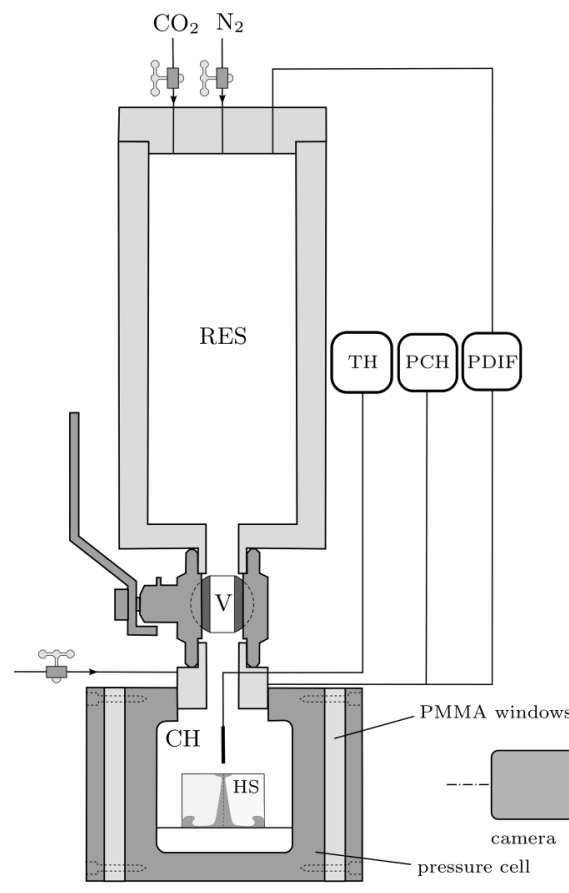

(b)

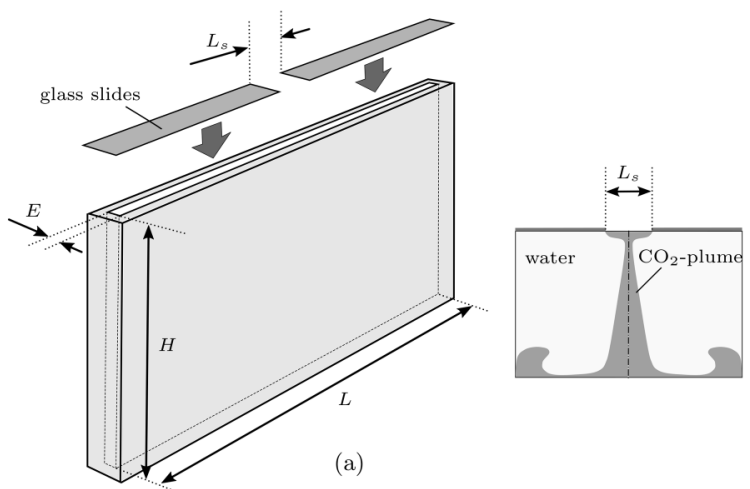

(a)

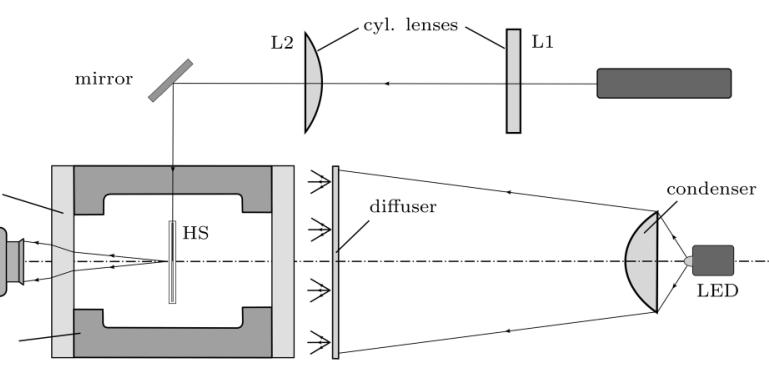

(c)

FIG. 1. Experimental setup. (a) Hele-Shaw cell and masking glass slides. (b) Side view of the setup. HS: Hele-Shaw cell; RES: reservoir; CH: pressure chamber; V: ball valve; PCH: pressure sensor; PDIF: differential pressure sensor; TH: thermocouple. (c) Top view of the PIV and $p \mathrm{H}$-sensitive dye visualization systems (see the text for further explanations).

dimensionless parameter of the problem. The similarities and discrepancies between numerical simulation and experiments are discussed in the final paragraph of the section (Sec. IV D). The concluding Sec. V summarizes the main results of the study and offers prospects for future work.

\section{PRESENTATION OF THE PROBLEM-MATERIALS AND METHODS}

\section{A. Experimental setup-Phenomenology of the dissolution}

The core of the experimental system consists of a Hele-Shaw cell of dimensions $L \times H \times E$ with $L=60, H=45$, and $E=0.5$ or $1 \mathrm{~mm}$. The cell is completely filled with water (density $\rho=998 \mathrm{~kg} \mathrm{~m}^{-3}$, viscosity $\mu \simeq 10^{-3} \mathrm{~Pa} \mathrm{~s}$, kinematic viscosity $\left.v=\mu / \rho\right)$ and placed vertically at the center of a pressure chamber as shown in Fig. 1. The upper open side of the Hele-Shaw cell is partially masked by means of two glass slides so that the free liquid/gas interface is left open over a region of width $L_{s} \ll L$ which will be referred to as a source in the following (with $L_{s}=4$ or $8 \mathrm{~mm})$.

The pressure chamber is equipped with four polymethylmethacrylate windows to allow for illumination - frontal white light illumination for $p \mathrm{H}$-sensitive dye visualization or side laser sheet illumination for particle image velocimetry (PIV). The pressure chamber is connected to a reservoir through a ball valve. The chamber, which is connected to a 300-bars nitrogen cylinder (BOC-Linde, U.K.), can be pressurized up to 10 bars (safety valve not shown in the figure). The reservoir is connected to the nitrogen cylinder and a 200-bars carbon dioxide cylinder, so that it can be filled with a mix $\left[\mathrm{N}_{2}+\mathrm{CO}_{2}\right]$ up to a total pressure of 10 bars. The main purpose of the setup is to increase the partial pressure of carbon dioxide in the chamber up to a given value $P_{\mathrm{CO}_{2}}$ from 1 to 5 bars and 
trigger a dissolution process of the carbon dioxide into the water through the free water/gas interface at the top of the Hele-Shaw cell. The role of the reservoir placed on top of the chamber is to prevent thermal effects due to the adiabatic compression which would occur in the case of an increase in the total pressure in the chamber.

Initially, the ball valve (volume $V_{V} \simeq 150 \mathrm{~cm}^{3}$ ) is closed. The chamber (volume $V_{C}=743 \mathrm{~cm}^{3}$ ) is filled with pure nitrogen at a total pressure $P$. Depending on the targeted partial pressure of carbon dioxide, the reservoir (volume $V_{R}=1570 \mathrm{~cm}^{3}$ ) is filled either with pure $\mathrm{CO}_{2}$ or with a mixture of $\mathrm{CO}_{2}$ (molar fraction $\omega$ ) and nitrogen (molar fraction $1-\omega$ ) at a slightly higher total pressure $P(1+\epsilon)$ with $\epsilon \sim 5 \%$. Upon opening of the valve, the carbon dioxide is convected into the chamber (due to the overpressure $\epsilon$ ) and a partial pressure of carbon dioxide,

$$
P_{\mathrm{CO}_{2}}=\frac{\omega V_{R}}{V_{R}+V_{V}+V_{C}} P
$$

is reached in the whole cavity after about $30 \mathrm{~s}$ without any large change in the total pressure (and, hence, no related thermal effects). Note that in the case where the reservoir is filled with pure $\mathrm{CO}_{2}$ $(\omega=1)$ and because the initial total pressure in the system before opening the valve cannot be smaller than one atmosphere, the lower bound of the accessible final partial pressure of $\mathrm{CO}_{2}$ is limited to 0.64 bars by the volume ratio $V_{R} /\left(V_{R}+V_{V}+V_{C}\right)$. Therefore, mixing nitrogen and carbon dioxide-i.e., $\omega<1$-becomes necessary to achieve smaller values of $P_{\mathrm{CO}_{2}}$. During the increase in $P_{\mathrm{CO}_{2}}$ and the subsequent plateauing period, the carbon dioxide starts dissolving in the liquid within the Hele-Shaw cell through the liquid/gas interface (i.e., the free surface of the liquid). Due to molecular diffusion, a layer of $\mathrm{CO}_{2}$-enriched water, denser than the water beneath it, starts growing just below the free surface. Almost immediately, this unstable configuration destabilizes and a solutal plume starts extending downwards. After a few minutes, once the $\mathrm{CO}_{2}$-enriched water has reached the bottom of the cell, a quasisteady regime settles during which the structure of the plume shows very little change.

\section{B. Measurement techniques}

PIV. PIV measurements require illumination by a laser sheet generated here from a green $520-\mathrm{nm}$ laser diode (Coherent StingRay Line Generator 123 1496, 35 mW, USA). The initial diverging laser Gaussian sheet is made parallel by means of a first F50 cylindrical lens (L1) placed at the focal distance of the laser outlet. A second F300 cylindrical lens (L2) is used to tune the thickness of the laser sheet in the region where the sample is located. Such an optical setup yields a Gaussian sheet with a thickness of $175 \pm 20 \mathrm{~nm}$ at the center of the Hele-Shaw cell. The finite thickness of the laser sheet induces a $15 \%$ drop of the measured velocity compared to the maximum velocity for $E=1 \mathrm{~mm}$. Such a bias has been corrected when comparing the theoretical and numerical values. The convection flows are made visible by adding tracer particles in the fluid. We use polystyrene spherical particles (1\% solid volume fraction, Bangs Laboratories, Inc., USA), $4.95 \mu \mathrm{m}$ in mean diameter, at very low concentration. The settling velocity of the particles, which is on the order of $1.8 \mu \mathrm{m} \mathrm{s}^{-1}$, is negligible compared to the velocity of the fluid during the plume growth and the subsequent steady regime. The PIV image sequences were recorded using a high-resolution $12 \mathrm{Mpx}$ color camera (Basler ace-acA4112-30uc, Basler, Germany) at frame rates lying in the range of 1-10 fps, converted into black/white 8-bit format and eventually processed using a crosscorrelation routine optimized for large velocity gradients [25]. The velocity measured at the center of the Hele-Shaw cell is then multiplied by a factor of $2 / 3$ in order to compare to the $y$-averaged velocity $\overline{\boldsymbol{u}}(x, z)$ modeled in the numerics.

$p H$-sensitive dye visualization. As dissolution of $\mathrm{CO}_{2}$ in water lowers the $p \mathrm{H}$ of the solution, the concentration field $C(x, y)$ of dissolved gas can be revealed by means of a $p \mathrm{H}$-sensitive dye. For this purpose, we use bromocresol purple (purchased from Sigma-Aldrich, USA and referred to as BCP in the following), the $p \mathrm{H}$ range of color change of which is [5.2 (yellow)-6.8 (purple)]. In all our experiments, the BCP concentration is $10^{-3} \mathrm{~mol} \mathrm{~L}^{-1}$. When used at such a low concentration, BCP 

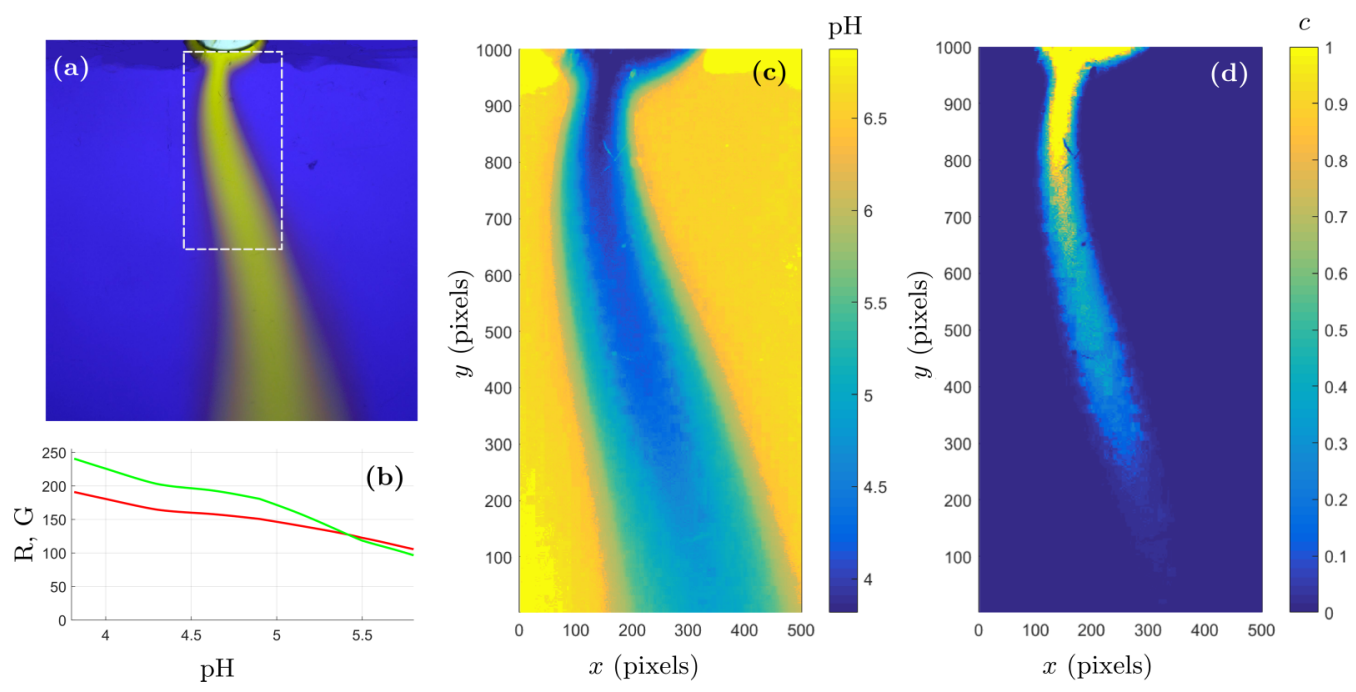

FIG. 2. (a) Raw image of the plume obtained for $L_{s}=4 \mathrm{~mm}$ and $P_{\mathrm{CO}_{2}}=1$ bar. (b) Color-to- $p \mathrm{H}$ calibration curves used to translate the raw image into a $p \mathrm{H}$ map. (c) Corresponding map $p \mathrm{H}(x, y)$ [rectangled region in (a)]. (d) Concentration map $C(x, z)$ extracted from (c) via the simplified relationship $p \mathrm{H}=\left(p C+p K_{a}\right) / 2$, where $p K_{a} \simeq 6.4$.

does not affect the gravity-induced plume dynamics, and, thus, the convective dissolution. For dye visualizations, the Hele-Shaw cell is illuminated frontally by means of a white light-emitting diode (LED) (MNWHL4, $740 \mathrm{~mW}$, Thorlabs, USA) and the camera used for PIV recording is also used to capture raw color maps, such as the one shown in Fig. 2(a) at a frame rate of $24 \mathrm{fps}$.

In order to extract quantitative values of $\mathrm{CO}_{2}$-concentration field, we first built a calibration color scale by measuring the uniform [red (R), green (G), and blue (B)] values for a set of $\mathrm{HCl}$ aqueous solutions. Such a calibration was performed in the Hele-Shaw, subsequently, used to observe the plume dynamics in the very same conditions of illumination and temperature. It turned out that the blue channel was not reliable and so was ruled out from the concentration extraction method. The calibration curves obtained for the $G$ and $R$ channels are shown in Fig. 2(b). These calibration curves were, subsequently, used to convert the raw color map into a $p \mathrm{H}$ map, the latter being, in turn, converted into a concentration map via the $p \mathrm{H}$-to-concentration relation $p \mathrm{H}=\left(p C+p K_{a}\right) / 2$ with $p K_{a}=-6.4$ and $p C=-\log _{10} C\left(C\right.$ being the concentration in carbon dioxide in $\left.\mathrm{mol} \mathrm{L}^{-1}\right)$.

The initial objective was to perform a full characterization of the concentration field in the plume. However, due to the strong nonlinearity (logarithmic) relationship between the carbon dioxide concentration and the $p \mathrm{H}$, we were only able to perform crude measurements of the plume lateral extension $\sigma$-defined as the width of the transverse concentration profile corresponding to a drop of the maximum amplitude by a factor of $1 / e$ - but not of the actual concentration amplitude, which was marred by about one order of magnitude of uncertainty. Note that performing a real quantitative assessment of the concentration amplitude would be an arduous task-see, e.g., Ref. [26]. The measurements of the plume width are shown for information in the very last figure of the article (Fig. 11).

It is worth noting that the white LED frontal illumination system used for $p \mathrm{H}$-sensitive dye visualization generated weak thermoconvective recirculation flows which, for the lowest partial pressure of carbon dioxide $\left(P_{\mathrm{CO}_{2}}=1 \mathrm{bar}\right)$, were no longer negligible compared to the velocity field associated with the gravitational convection. In Figs. 2 and 12, the steady curvature of the plume axis is due to the effect of such flows and does not reflect the presence of any unsteadiness, such as meandering instabilities. Thermoconvective flows were also visible when the system was 
illuminated from the left by a green laser sheet (PIV measurements), but the effect was then much less important; in general, the thermoconvective perturbations had minimal impact on the PIV measurements ( $\sim 5 \%$ of additional uncertainty on the vertical velocity amplitude).

\section{Governing equations}

The dynamics of the solutal plume is governed by the Navier-Stokes equation along with the continuity equation and the advection-diffusion equation for the carbon dioxide concentration. In order to provide a generic picture of the convective dissolution process, we use in the following the dimensionless forms of these equations. To this end, we choose $L_{s}, D / L_{s}$, and $L_{s}^{2} / D$ as, respectively, the typical length, velocity, and timescales of the problem, $\left(D=1.76 \pm 0.04 \times 10^{-9} \mathrm{~m}^{2} \mathrm{~s}^{-1}\right.$ being the molecular diffusivity of the carbon dioxide in water). The concentration in carbon dioxide is made dimensionless by the concentration at saturation $K_{H} P_{\mathrm{CO}_{2}}$-where $K_{H}$ is the Henry's constant for the dissolution of carbon dioxide in water. We also consider the density at the liquid-gas interface as a linear function of the concentration and define the solutal expansion coefficient as $\alpha=\left(1 / \rho_{0}\right)(\partial \rho / \partial C)$. Thus, the density difference between pure and $\mathrm{CO}_{2}$-enriched water is $\Delta \rho=\rho_{0} \alpha K_{H} P_{\mathrm{CO}_{2}}$. The value of the product $\alpha K_{H}$ has been measured to be $0.004 \pm 0.001 \mathrm{MPa}^{-1}$ by Hebach et al. [27], but their results were marred by a large uncertainty in the low-pressure domain, which, therefore, leads to a large uncertainty (on the order of $25 \%$ ) in the determination of the Rayleigh number. Based on the aforementioned typical scales, the dimensionless forms of the governing equations are for the flow,

$$
\frac{\mathrm{Da}}{\mathrm{Sc}}\left[\frac{\partial \boldsymbol{u}}{\partial t}+(\boldsymbol{u} \cdot \nabla) \boldsymbol{u}\right]=-\nabla p+\operatorname{Da} \nabla^{2} \boldsymbol{u}+\operatorname{Ra} c \boldsymbol{z}, \quad \nabla \cdot \boldsymbol{u}=0,
$$

and for solute transport,

$$
\frac{\partial c}{\partial t}+(\boldsymbol{u} \cdot \nabla) c=\nabla^{2} c .
$$

In these equations, the Rayleigh, Darcy, and Schmidt numbers are, respectively, defined as

$$
\mathrm{Ra}=\frac{\Delta \rho g \kappa L_{s}}{\mu D}=\frac{\alpha \rho_{0} K_{H} P_{\mathrm{CO}_{2} g \kappa L_{s}}}{\mu D}, \quad \mathrm{Da}=\frac{\kappa}{L_{s}^{2}} \quad \text { and } \quad \mathrm{Sc}=\frac{\nu}{D},
$$

where $g$ is the gravity acceleration and $\kappa=E^{2} / 12$ is the equivalent permeability of the Hele-Shaw cell.

In order to further simplify the problem, we make the so-called Brinkman assumption that the flow velocity profile is parabolic across the cell thickness and has no component in the $y$ direction, which can be written

$$
\boldsymbol{u}(x, y, z)=\frac{3}{2} \overline{\boldsymbol{u}}(x, z)\left[1-\left(\frac{2 y}{e}\right)^{2}\right]
$$

where the $y$-averaged velocity is defined as

$$
\overline{\boldsymbol{u}}(x, z)=\frac{1}{e} \int_{-e / 2}^{e / 2} \boldsymbol{u}(x, y, z) d y, \quad \text { with } \quad e=\frac{E}{L_{s}} .
$$

After taking the average over the cell's thickness of Eqs. (2) and (3), one obtains the bi-dimensional forms of the governing equations,

$$
\begin{gathered}
\frac{\mathrm{Da}}{\mathrm{Sc}}\left[\frac{\partial \overline{\boldsymbol{u}}}{\partial t}+\frac{6}{5}\left(\overline{\boldsymbol{u}} \cdot \nabla_{\|}\right) \overline{\boldsymbol{u}}\right]=-\nabla_{\|} \bar{p}+\left[\mathrm{Da} \nabla_{\|}^{2}-1\right] \overline{\boldsymbol{u}}+\operatorname{Ra} \bar{c} z, \\
\frac{\partial \bar{c}}{\partial t}+\left(\overline{\boldsymbol{u}} \cdot \nabla_{\|}\right) \bar{c}=\nabla_{\|}^{2} \bar{c}
\end{gathered}
$$


where $\nabla_{\|}=\left(\partial_{x}[\cdots], \partial_{z}[\cdots]\right)$ and $\nabla_{\|}^{2}=\partial_{x}^{2}+\partial_{z}^{2}$, respectively, are the bi-dimensional gradient and the Laplace operators and where $\bar{p}$ is the $y$-averaged pressure. In the following, the vertical $z$ component of the $y$-averaged velocity is denoted by $\bar{w}$.

Note that considering an averaged form of the nonlinear term as written in Eq. (8) is not self-evident. In the present case, there is no concentration front propagating downwards since we deal with a quasisteady situation where the plume has already reached the bottom of the cell. Therefore, the concentration gradients are mostly transverse; as the flow can be approximated as a parallel flow along the $z$ direction, the only velocity gradients are transverse as well. So the concentration gradients are very weak in the direction of the velocity gradients, and, therefore, Taylor-Aris dispersion does not occur. That is the reason why the effective Taylor-Aris dispersion is ignored when averaging the advection-diffusion equation for the concentration. The flat transverse concentration profile assumption has been tested using three-dimensional numerical simulations at different locations where concentration $(x, z)$ gradients were substantial, and it turned out that the concentration was actually independent of the $y$ coordinate.

Equations (7) and (8) must be complemented by a suitable set of boundary conditions. In practice, a no-slip condition $\overline{\boldsymbol{u}}=\mathbf{0}$ applies at the walls whereas a zero tangential stress condition is to be considered at the free interface. However, the presence of impurities at the free surface blocks the tangential velocity (as shown in Ref. [22]) so that a no-slip boundary condition at the free surface better represents the experimental result. As shown in the experimental part, the free interface is not strictly flat and a bi-dimensional meniscus appears as time passes due to slow evaporation. However, experimental and numerical results have shown to be almost independent of the Bond number so that the meniscus curvature and the corresponding locally baroclinic situation at the interface are ignored when it comes to interpreting the obtained numerical scaling laws (see Sec. IV C). Finally, the concentration is supposed to take its saturation value at the interface-i.e., $\bar{c}=1$ at the interface. In the following, the bars used to refer to the space-averaged quantities are dropped to make the notations less cumbersome. However, we keep the $\nabla_{\|}$notation to remind the reader that the governing equations are depth averaged.

\section{Numerical methods}

Since we were not able to investigate experimentally more than one order of magnitude in Rayleigh, an extensive numerical investigation of the plume dynamics has been performed by means of a commercial code (COMSOL MULTIPHYSICS ()). Equations (7) and (8) are solved numerically in their dimensionless forms together with no-slip boundary conditions at the solid walls and at the interface. The whole fluid region is left-right symmetric since the source is located halfway from the lateral vertical walls, therefore, only half of the domain is considered from $x=0$ to 5 and $z=0$ to 5 with a symmetry condition on the left vertical boundary (symmetry axis of the plume). An expanded view of the meshing in the vicinity of the source is shown in Fig. 3(a). The mesh has been refined with a mesh size equal to $\Delta x=0.01$ underneath the liquid interface, which lies between $x=0$ and $x=0.5$ and along the axis of the plume. The numerical scheme uses a finite element approach with P2 + P1 discretization for the velocity, pressure, and cubic discretization for the concentration. This leads to $3 \times 10^{5}$ degrees of freedom for a mesh with 20000 elements. As mentioned in the previous section, the shape of the meniscus plays almost no role in the plume dynamics. However, in order to ensure lateral stability of the plume, especially in the early times of the destabilization process, the liquid/gas interface has been designed with a slight slope (2\%) oriented towards the axis, which is visible in Fig. 3(a). A convergence study has been performed at $\mathrm{Ra}=10^{6}$ and $\mathrm{Da}=1$ to find the optimal mesh size as shown in Fig. 3(b) where the vertical velocity in the quasisteady regime on the symmetry axis (at $z=1 / 2$, dimensionless units) is plotted with respect to the mesh size $\Delta x$. It is clear that the plume velocity is already converged for a mesh size as large as ten times the mesh size chosen for all simulations. For the largest Rayleigh number the flow became unsteady so that a selective frequency damping [(SFD), see Ref. [28]) was applied after the onset of the plume. An example is shown for $\mathrm{Ra}=10^{5}$ and $\mathrm{Da}=0.01$ in Fig. 3(c) 

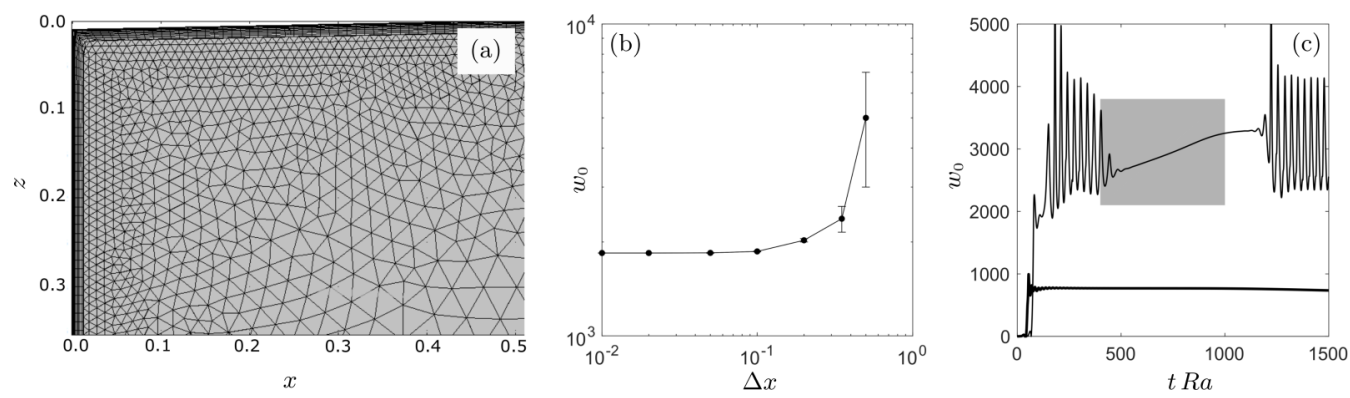

FIG. 3. (a) Expanded view of the bi-dimensional mesh in the vicinity of the source (b) convergence of the simulation with respect to the mesh size $\Delta x$ for $\mathrm{Ra}=10^{6}$ and $\mathrm{Da}=1$. (c) Time profile of the velocity for $\mathrm{Ra}=10^{4}$ (thin line) and $\mathrm{Ra}=10^{5}$ (thick line) at $\mathrm{Da}=0.01$.

where the SFD (applied between $t \mathrm{Ra}=400$ and 900) damps the oscillations of the plume velocity. When the SFD is switched off, the plume destabilizes again but after a quasisteady regime which is sufficiently long for the determination of the (ideal) steady plume velocity.

\section{EXPERIMENTAL RESULTS}

The maximum vertical velocity $w_{0}$ along the transverse $x$ direction at $z=1$ (one source size below the bottom of the meniscus) is plotted in Fig. 4(b) as a function of the dimensionless time $t$. After the starting plume has reached its terminal velocity, which occurs before the front of the plume has touched the bottom of the cell, $w_{0}$ plateaus at a value which remains unchanged during what can be considered as a steady regime. Later, as expected, once the whole fluid is close to being saturated in carbon dioxide, the plume decreases in strength and eventually vanishes; such a decreasing regime is not represented in Fig. 4(b).

A typical velocity field $\langle w\rangle$ resulting from the time averaging of all the instantaneous velocity fields over the entire duration of the steady regime is shown in Fig. 4(a) together with a raw image where the meniscus is clearly visible. From a transverse $x$ profile of the vertical component of this averaged velocity field (i.e., at a given altitude $z$ ), the maximum $\left\langle w_{0}\right\rangle$ and the lateral extension $\left\langle\sigma_{w}\right\rangle$ of the profile (width of the profile corresponding to a drop of the amplitude by a factor of $1 / e$ ) can be defined without ambiguity. The transverse profile shown in Fig. 4(c), which corresponds to the altitude $z=1$ of the averaged field presented in Fig. 4(a), has been obtained for Da $=5.2 \times 10^{-3}$ and $\mathrm{Ra}=2973\left(E=1, L_{s}=4 \mathrm{~mm}, P_{\mathrm{CO}_{2}}=4 \mathrm{bars}\right)$ but is representative of what is observed in the entire $(\mathrm{Ra}, \mathrm{Da})$ domain explored experimentally. Close to its maximum, the transverse velocity profile can be reasonably fitted by a function of the form $\left\langle w_{0}\right\rangle\left[\cosh \left(x /\left\langle\sigma_{w}\right\rangle\right)\right]^{-2}$ [thin dashed line in Fig. 4(c)] characteristic of a low Darcy number bi-dimensional plume (see Sec. IV A); it decreases as $\exp \left(-x /\left\langle\sigma_{w}\right\rangle\right)$ for $x \rightarrow \infty$. However, at a distance of the plume axis on the order of $2\left\langle\sigma_{w}\right\rangle$, the profile departs from that exponential decay and gets closer to a Gaussian curve of standard deviation $\sqrt{2}\left\langle\sigma_{w}\right\rangle$ [dotted curve in Fig. 4(a)], i.e., at large $x$ the profile scales as $\exp \left[-\left(x /\left\langle\sigma_{w}\right\rangle\right)^{2} / 2\right]$.

The maximum velocity on the plume axis $\left\langle w_{0}\right\rangle$ is plotted in Fig. 4(d) as a function of the altitude $z$. This profile first increases up to $z=0.5$ and then roughly plateaus until $z=4$. Note that there is a $25 \%$ local decrease in the velocity magnitude in the vicinity of $z=1$ which is not an experimental artifact but rather corresponds to an actual slow down of the plume by the recirculating flow. The corresponding width $\left\langle\sigma_{w}\right\rangle$ [lateral extension of the velocity profile in Fig. 4(c)] is plotted in Fig. 4(e) with respect to the altitude. It is an increasing function of the vertical position roughly proportional to $z^{1 / 3}$. Note that such a scaling law is nothing more than indicative considering the reduced range in $z$ used to retrieve it. Indeed, this plume width can only be defined for $z>0.5$ since the profile has two maxima for small $z$. 

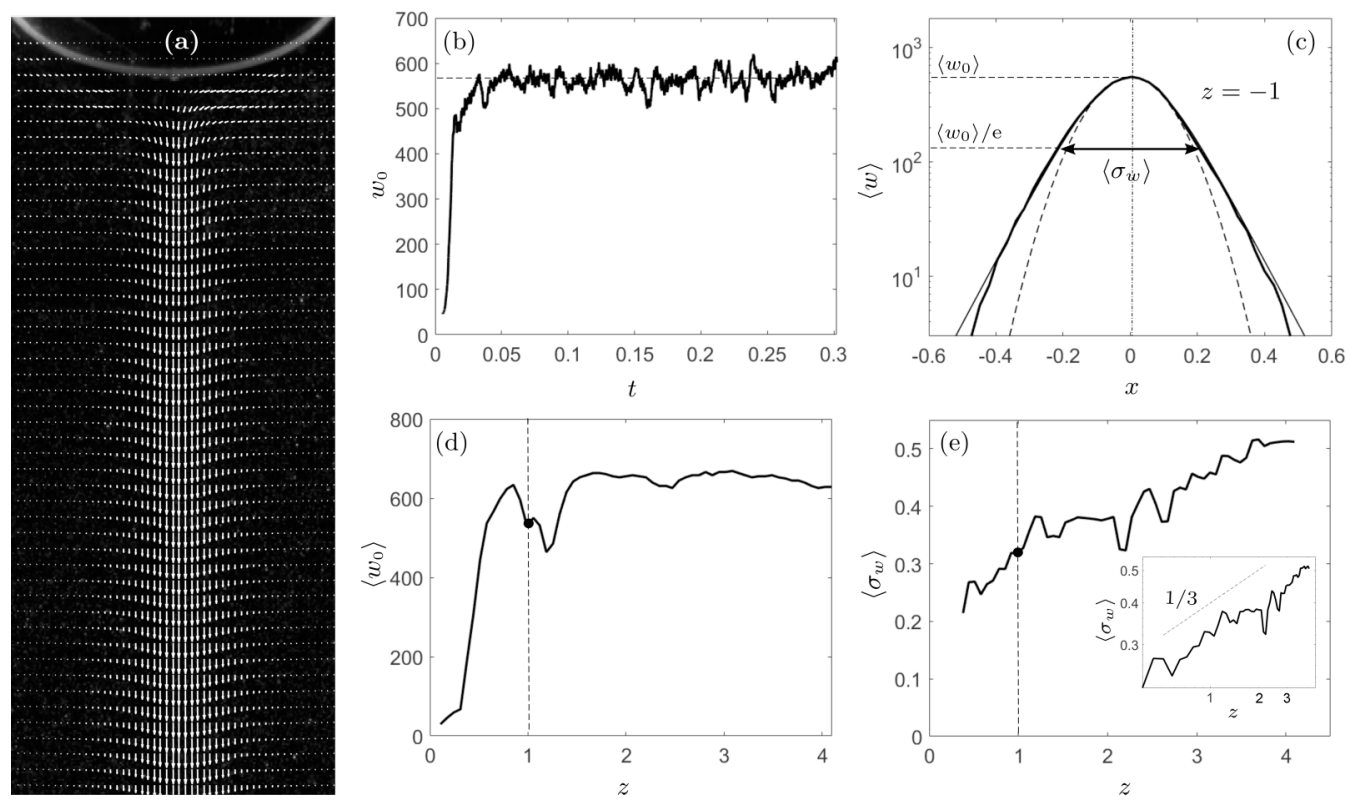

FIG. 4. (a) Velocity field $\langle w\rangle$ averaged over the period of time (0.05-0.025) for the dimensionless parameters $\mathrm{Da}=5.2 \times 10^{-3}$ and $\mathrm{Ra}=2973\left(E=1, L_{s}=4 \mathrm{~mm}, P_{\mathrm{CO}_{2}}=4\right.$ bars $)$. The space scale is provided by the width of the image which is equal to 1 (width of the source in dimensionless units). (b) Time profile of the maximum velocity measured on transverse profiles at $z=1$. (c) Average transverse profile $\langle w\rangle$ measured at $z=1$ (thick solid line). The fitting function of the form $\left\langle w_{0}\right\rangle\left[\cosh \left(1.085 x /\left\langle\sigma_{w}\right\rangle\right)\right]^{-2}$ is plotted with a thin solid line, whereas the Gaussian function of same curvature on the axis is plotted with a dashed line. (d) Axial $z$-profile $\left\langle w_{0}\right\rangle$ of the maximum velocity obtained from the averaged field $\langle w\rangle$ shown in (a). (e) Axial $z$ profile of the width $\left\langle\sigma_{w}\right\rangle$.

The quantities $\left\langle w_{0}\right\rangle$ and $\left\langle\sigma_{w}\right\rangle$ have been measured at $z=1$ for different combinations of the dimensionless numbers $\mathrm{Ra}$ and $\mathrm{Da}-$ i.e., for all sets of physical parameters $E, L_{s}$, and $P_{\mathrm{CO}_{2}}$ reported in Table I. The obtained experimental data are plotted together with the numerical results in the last and central figure of the article (Fig. 11) and commented in the Discussion section in light of the numerical simulations presented in the next section and of the scaling laws derived in Sec. IV C.

\section{NUMERICAL INVESTIGATION}

As shown in Table I, the explored range of Rayleigh numbers lies over no more than one order of magnitude, and only two values of Darcy numbers are considered in the experiments. In order to have a broader picture of the plume dynamics and to more accurately characterize the dependence of the two quantities $\left\langle w_{0}\right\rangle$ and $\left\langle\sigma_{w}\right\rangle$ on the dimensionless numbers, a series of numerical simulations has been performed for the values of Da and Ra listed in Table II. For each Darcy number, the

TABLE I. Values of the Rayleigh number corresponding to the different values of the physical parameters $P_{\mathrm{CO}_{2}}$ and $L_{s}$ in the experiments. In all cases, the thickness of the cell is $E=1 \mathrm{~mm}$.

\begin{tabular}{lccccc}
\hline \hline & $P_{\mathrm{CO}_{2}}=1$ bar & $P_{\mathrm{CO}_{2}}=2$ bars & $P_{\mathrm{CO}_{2}}=3$ bars & $P_{\mathrm{CO}_{2}}=4$ bars & $P_{\mathrm{CO}_{2}}=5$ bars \\
\hline$L_{s}=4 \mathrm{~mm}\left(\mathrm{Da}=5.2 \times 10^{-3}\right)$ & 743 & 1486 & 2230 & 2973 & 3715 \\
$L_{s}=8 \mathrm{~mm}\left(\mathrm{Da}=1.3 \times 10^{-3}\right)$ & 1486 & 2973 & 4459 & 5946 & 7432 \\
\hline \hline
\end{tabular}


TABLE II. Values of the Rayleigh number considered for all values of the Darcy number in the numerical simulations. The simulations where SFD has been used to stabilize the plume are specified by stars ( $\star$ ).

\begin{tabular}{ll}
\hline \hline $\mathrm{Da}$ & \multicolumn{1}{c}{$\mathrm{Ra}$} \\
\hline $10^{-5}$ & {$\left[30,10^{2}, 3 \times 10^{2}, 10^{3 \star}\right]$} \\
$10^{-4}$ & {$\left[30,10^{2}, 3 \times 10^{2}, 10^{3 \star}, 3 \times 10^{3 \star}\right]$} \\
$10^{-3}$ & {$\left[30,10^{2}, 10^{3 \star}, 3 \times 10^{3 \star}\right]$} \\
$10^{-2}$ & {$\left[30,10^{2}, 10^{3}, 10^{4}, 10^{5 \star}\right]$} \\
$10^{-1}$ & {$\left[10^{2}, 10^{3}, 10^{4}, 10^{5 \star}, 10^{6 \star}\right]$} \\
1 & {$\left[10^{3}, 10^{4}, 10^{5}, 10^{6}, 10^{7}\right]$} \\
\hline \hline
\end{tabular}

minimum Rayleigh number corresponds to a case where the width of the plume becomes on the order of 1 and the maximum Rayleigh number corresponds to a case where the plume is strongly unstable, so the steady regime cannot be reached even by resorting to the SFD.

\section{A. Darcy regime}

We first investigate the low Darcy number regime. In Fig. 5(a), the velocity and concentration fields obtained for $\mathrm{Da}=10^{-3}$ and $\mathrm{Ra}=10^{3}$ are superimposed on each other. In such a regime the depth-averaged Navier-Stokes Eq. (7) simplifies to

$$
-\nabla_{\|} p-\boldsymbol{u}+\mathrm{Ra} c z=\mathbf{0},
$$

since inertial and Brinkman terms can be ignored. This Darcy equation considered together with the steady version of Eq. (8) has been solved in the case of a point source by Wooding [23], who showed that the solution could be written in the form (see Appendix B for full derivation of the dimensionless form)

$$
w=\operatorname{Ra} c=\frac{2}{3}\left(\frac{9 \mathrm{Nu} \mathrm{Ra}}{16}\right)^{2 / 3} z^{-1 / 3} \cosh ^{-2}\left[\frac{1}{3}\left(\frac{9 \mathrm{Nu} \mathrm{Ra}}{16}\right)^{1 / 3} \frac{x}{z^{2 / 3}}\right],
$$
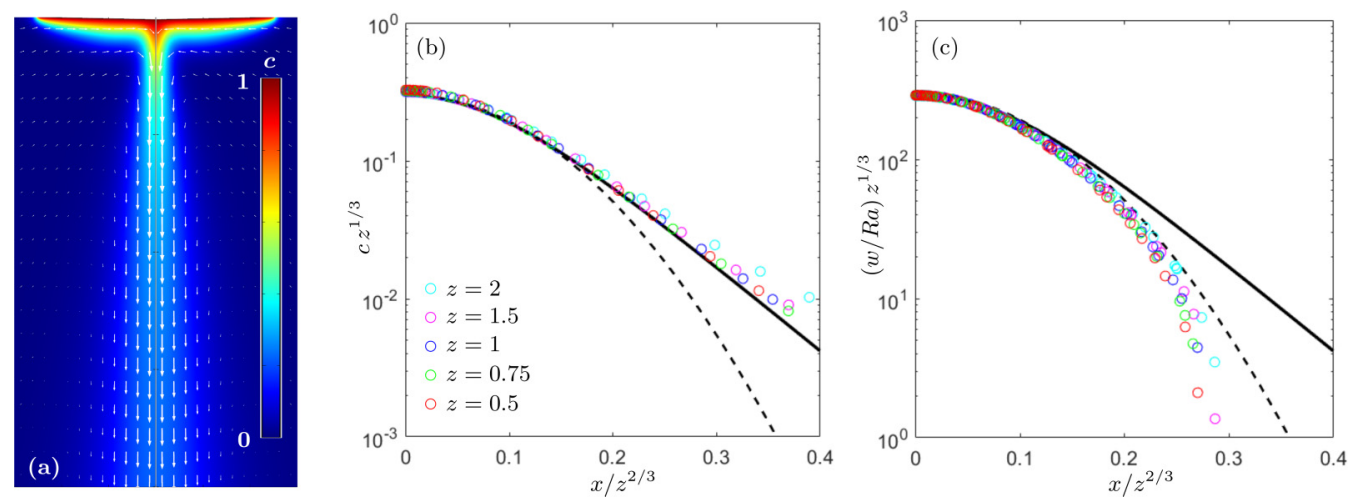

FIG. 5. (a) Numerical velocity and concentration fields obtained for $\mathrm{Ra}=10^{3}$ and $\mathrm{Da}=10^{-4}$. (b) Normalized transverse profile of the concentration as a function of the normalized $x$ coordinate, based on solution (10). (c) Normalized transverse profile of the vertical component of the velocity as a function of the normalized $x$ coordinate, based on solution (10). In both (b) and (c) the numerical data (open circles) correspond to the fields shown in (a), and the theoretical profiles corresponding to Eq. (10) are plotted as a solid line with $\mathrm{Nu}=16.8$ obtained from (11). The Gaussian function of same curvature on the axis is plotted as a dashed line. 

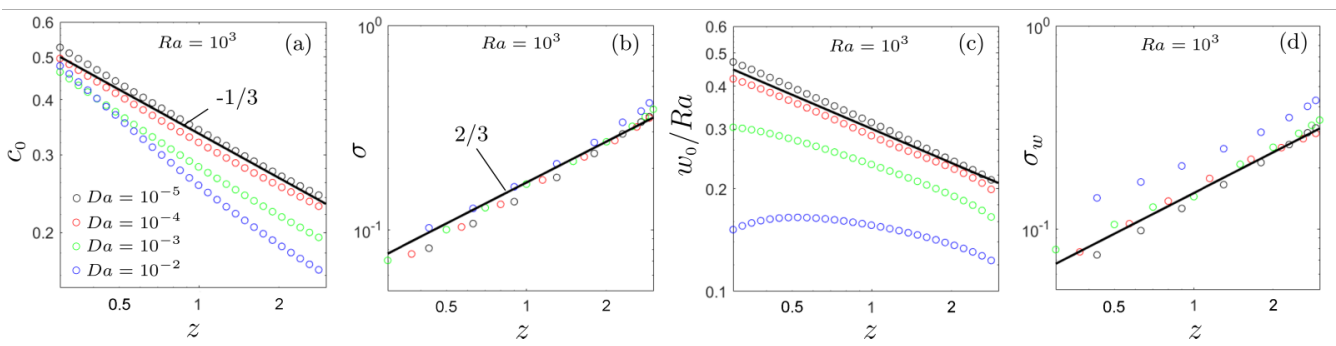

FIG. 6. (a) Axial $z$ profile of the maximum concentration $c_{0}$. (b) Width of the numerical concentration profile $\sigma$ as a function of $z$. (c) Axial $z$ profile of the maximum velocity $w_{0}$. (d) Width of the numerical transverse profile of the vertical velocity component $\sigma_{w}$ as a function of $z$. The numerical data are plotted for $\mathrm{Ra}=10^{3}$ and four different values of the Darcy number (open colored symbols). The theoretical scalings (solid black line) correspond to the low Darcy solution (10) derived by Wooding [23] in the case of a line source.

with

$$
\mathrm{Nu}=\int_{-\infty}^{+\infty} c w d x
$$

The Nusselt number depends neither on the $z$ coordinate nor on time since we are dealing with a stationary configuration. As suggested by this theoretical solution, five transverse profiles at different altitudes of the concentration $c$ renormalized by $z^{-1 / 3}$ are plotted as a function of the renormalized coordinate $x / z^{2 / 3}$ in Fig. 5(b). It is striking to see that all the profiles collapse extremely well, indicating that the scalings of (10) are well recovered. The theoretical profile (10) derived by Wooding [23] for a line source is plotted as a solid line for a Nusselt number $\mathrm{Nu}=16.8$ measured from the numerics. The numerical concentrations match the theoretical profile fairly well, showing an exponential decrease at large $x$, which is characteristic of this low Darcy solution. The Gaussian profile of the same curvature at maximum concentration is shown in Fig. 5(b) to emphasize the difference to Wooding's solution [23]. The $z$ position of the line source, which is not defined in the case of an extended source (unless a full theoretical analysis is performed as previously performed by Whittaker and Lister [21] in the case of an axisymmetric plume generated from an extended source) is taken at $z=0$ (bottom of the meniscus).

Figure $5(\mathrm{c})$ presents the corresponding transverse profiles of velocity renormalized by $z^{-1 / 3}$ as a function of the renormalized coordinate $x / z^{2 / 3}$. As for the concentration, there is an excellent collapse of all numerical data, confirming the scalings laws of Wooding's solution [23]. As in case of the concentration, the numerical velocity's transverse profile is close to the theoretical low Darcy (point source) prediction in the vicinity of its maximum value (solid line). It should be noted that the Nusselt number, which is the only unknown parameter of solution (10), is the same as the one used for the concentration. However, the experimental data depart from Wooding's solution [23] at large $x$ to come closer to the Gaussian profile. Actually, the numerical velocity profile shows a reversal of the velocity profile at sufficiently long distances, corresponding to an (expected) upwards recirculating flow also visible in the experiments.

In order to check the dependence on the Darcy number, four characteristics of the plume are plotted in Fig. 6 as a function of $z$ for Da increasing from $10^{-5}$ to $10^{-2}$. At low Darcy numbers, these plots confirm that the maximum concentration $c_{0}$ and the maximum velocity $w_{0} /$ Ra scale as $z^{-1 / 3}$ whereas the width of the concentration profile $\sigma$ (defined as the width at amplitude $c_{0} / e$ ) and the width $\sigma_{w}$ of the velocity profile (defined as the width at amplitude $w_{0} / e$ ) scale as $z^{2 / 3}$, in excellent agreement with Wooding's solution [23]. For the low Darcy numbers ( $\mathrm{Da}=10^{-4}$ and $\left.10^{-5}\right)$ not only the exponents, but also the prefactors of these scaling laws are independent of the Darcy number. This comes from the fact that Da does not appear in the Darcy equation (9). However, as the Darcy number becomes larger, the numerical solution becomes Darcy dependent in a manner especially 

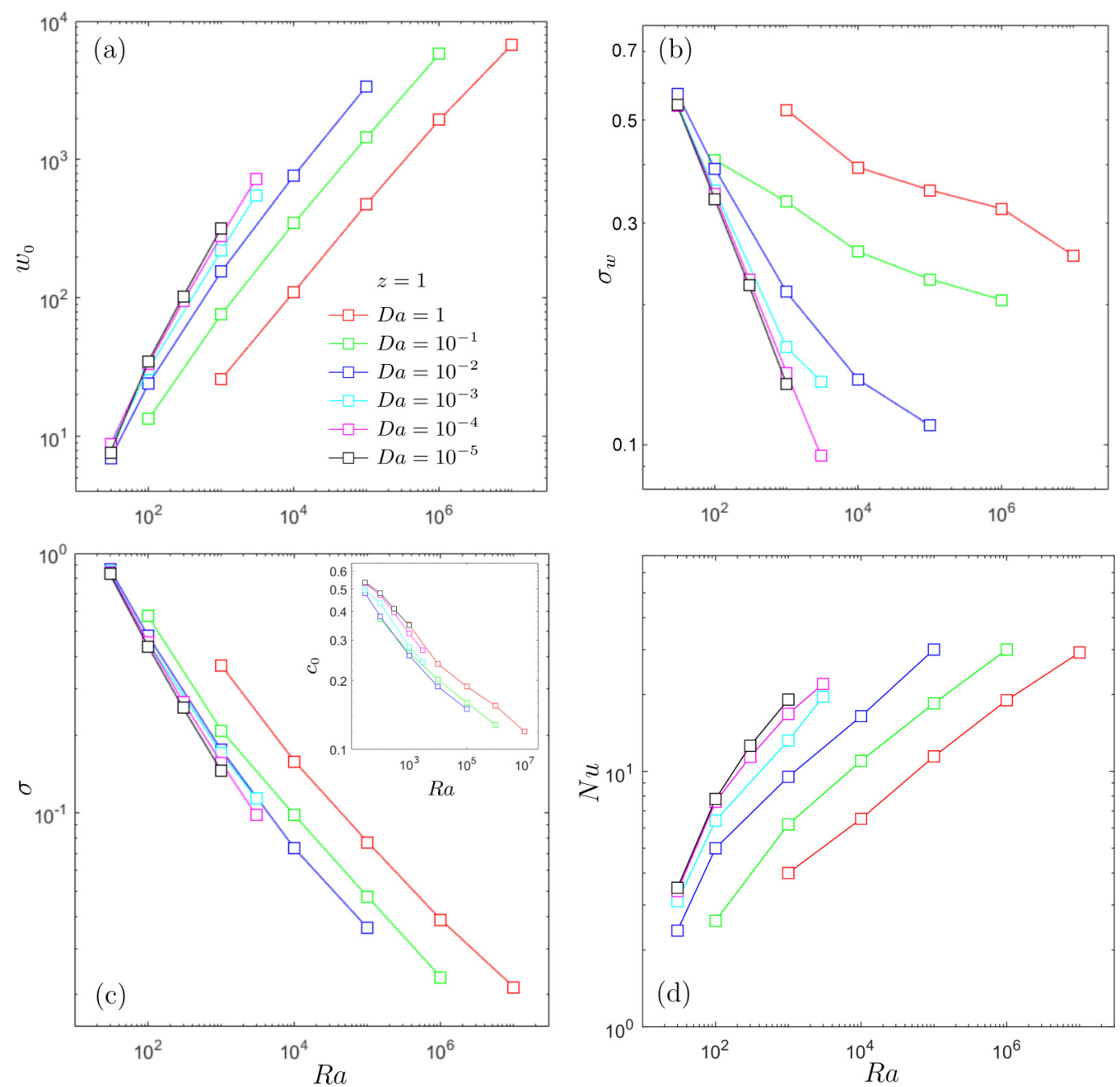

FIG. 7. (a) Maximum of the vertical velocity component (on the plume axis), (b) width of the transverse velocity profile, (c) width of the concentration profile (the maximum value of the transverse concentration profile is shown in the inset), and (d) Nusselt number as functions of the Rayleigh number. In (a)-(d) each quantity (extracted from the numerical calculation at $z=1$ ) is plotted for all the values of the Darcy number listed in Table II.

prominent for the quantities $c_{0}$ and $w_{0} / \mathrm{Ra}$. The effect is extremely pronounced for the maximum vertical velocity component, which becomes almost flat for $\mathrm{Da}=10^{-2}$. This is consistent with the experimental observation at $\mathrm{Da}=5.2 \times 10^{-3}$ and $\mathrm{Ra}=2973$ (see Fig. 4) although the maximum of the velocity profile is located at $z=2$ in the experiments whereas it is located at $z=0.5$ in the numerics. The exponent of the width $\sigma_{w}$ tends to decrease as Da increases, which can also explain the exponent $1 / 3$ obtained in the experiments rather than $2 / 3$ for the lowest Darcy numbers.

The dependence on the Rayleigh number is illustrated in Fig. 7 where the aforementioned characteristics taken at $z=1$ and the Nusselt number are plotted as a function of Ra. For small Darcy numbers $\left(\mathrm{Da}=10^{-5}, 10^{-4}, 10^{-3}\right)$, all the quantities collapse reasonably well on a single curve corresponding to the theoretical prediction of Eq. (10), whereas a clear dependence on the Darcy number is visible for larger values $\left(\mathrm{Da}=10^{-2}, 10^{-1}, 1\right)$. For low Darcy numbers, the Nusselt number seems to scale as $\mathrm{Ra}^{1 / 2}$. This scaling is interesting because it permits to maintain the 

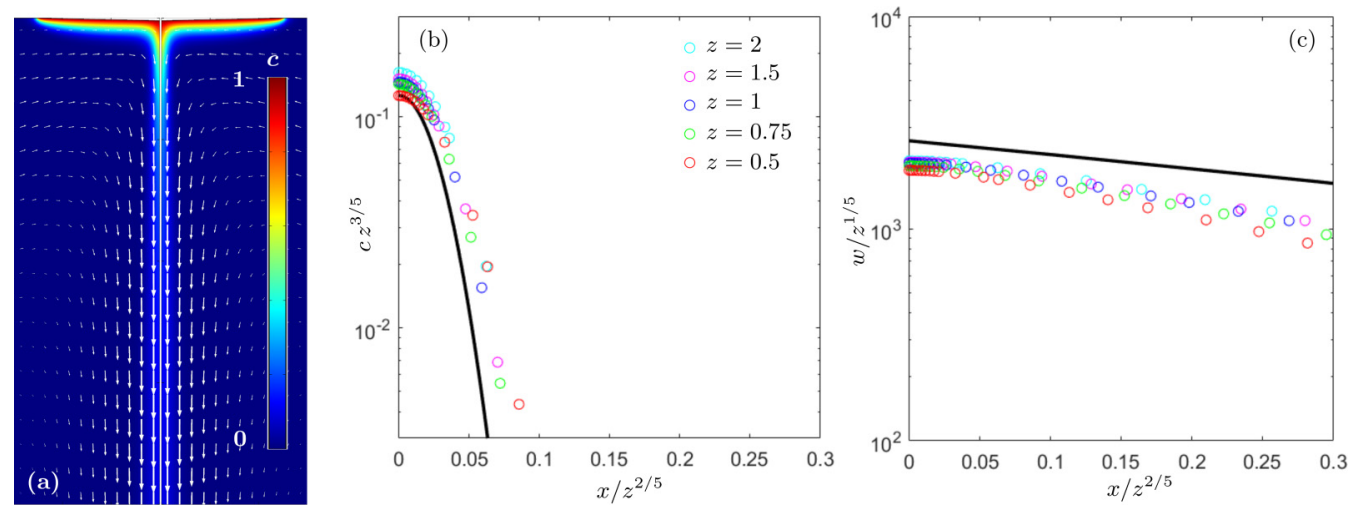

FIG. 8. (a) Numerical velocity and concentration fields obtained for $\mathrm{Ra}=10^{6}$ and $\mathrm{Da}=1$. (b) Normalized transverse profile of the concentration as a function of the normalized $x$ coordinate, based on solution (13). (c) Normalized transverse profile of the vertical component of the velocity as a function of the normalized $x$ coordinate, based on solution (13). In both (b) and (c) the numerical data (open circles) correspond to the fields shown in (a) and the theoretical profiles [Eqs. (13)] are plotted with $\mathrm{Nu}=19$ as given by (11) in the numerics.

concentration $c_{0}$ on the order of one at $z=1$ in Woodings's solution [23] (10). This scaling of the Nusselt number implies that the maximum velocity scales as $\mathrm{Ra}$, and that the widths $\sigma$ and $\sigma_{w}$ scale as $\mathrm{Ra}^{-1 / 2}$, which is consistent with the numerical findings. These scalings will be discussed in more details in Sec. IV C.

To conclude, the plumes match very well the point source solution of Wooding [23] for the lowest Darcy numbers but depart from this model as Da increases and get close to 1 . It indicates the existence of another regime for large Darcy numbers which is studied in the next section.

\section{B. Beyond the Darcy regime: Brinkman regime}

In Fig. 8(a), the velocity and concentration fields obtained for $\mathrm{Da}=1$ and $\mathrm{Ra}=10^{6}$ are superimposed on each other. It is clear in this regime that the plume's velocity remains significant over a large $x$ range whereas the solute remains confined in a very thin region around the axis. Furthermore, the velocity keeps increasing as $z$ increases, in contrast to the low Darcy case where it was decreasing. In this regime the Darcy term $-\mathbf{u}$ in the $y$-averaged Navier-Stokes Eq. (7) becomes negligible in comparison to the so-called Brinkman term $\mathrm{Da} \nabla_{\|}^{2} \boldsymbol{u}$. Indeed, the $x$ gradients are so large that the Brinkman term becomes dominant if the Darcy number is on the order of 1 . In this Brinkman regime, the depth-averaged Navier-Stokes Eq. (7) simplifies to

$$
\left(\boldsymbol{u} \cdot \boldsymbol{\nabla}_{\|}\right) \boldsymbol{u}=(5 / 6) \operatorname{Sc}\left[-\nabla_{\|} p+\nabla_{\|}^{2} \boldsymbol{u}+(\mathrm{Ra} / \mathrm{Da}) c z\right] .
$$

This viscous equation, considered together with the steady version of Eq. (8), has been solved in the case of a line source by Spalding and Cruddace [24]. He showed that the solution could be written in the limit of large Schmidt numbers (see Appendix C for full derivation) as

$w=\left(\frac{\sqrt{\mathrm{Sc} N u \mathrm{Ra}}}{\mathrm{Da}}\right)^{2 / 5}\left(\frac{5 z}{6}\right)^{1 / 5} g^{\prime}(\eta)$ and $c=\frac{1}{2 \sqrt{\pi g_{0}^{\prime}}}\left(\frac{\mathrm{Nu}^{4} \mathrm{Ra}}{\sqrt{\mathrm{ScDa}}}\right)^{1 / 5}\left(\frac{5 z}{6}\right)^{-3 / 5} \exp \left(\frac{-\mathrm{Sc} g_{0}^{\prime} \eta^{2}}{4}\right)$,

where

$$
\eta=\left(\frac{36 \mathrm{Nu} \mathrm{Ra}}{25 \mathrm{Sc}^{2} \mathrm{Da}}\right)^{1 / 5} \frac{x}{z^{2 / 5}} \text { and } \quad g_{0}^{\prime} \simeq 0.9335
$$



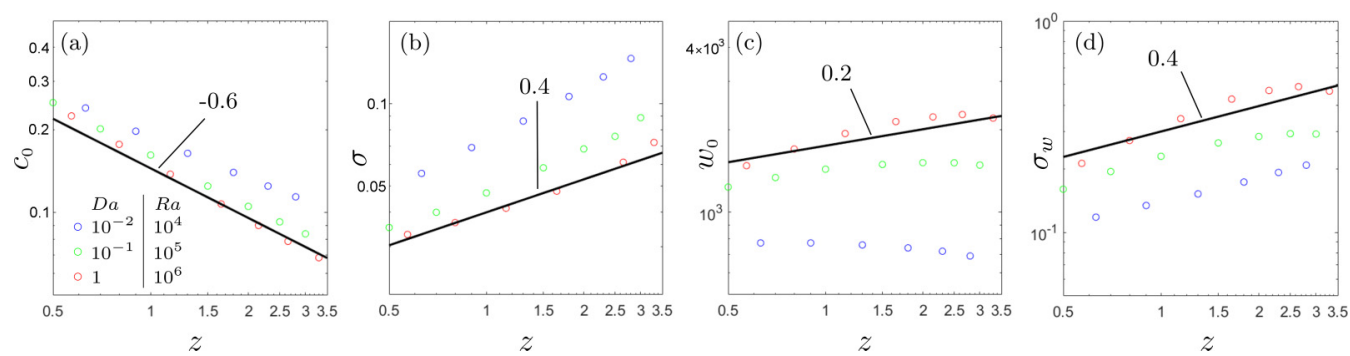

FIG. 9. (a) Axial $z$ profile of the maximum concentration $c_{0}$. (b) Width of the numerical transverse concentration profile $\sigma$ as a function of $z$. (c) Axial $z$ profile of the maximum velocity $w_{0}$. (d) Width of the numerical transverse velocity profile $\sigma_{w}$ as a function of $z$. The numerical data are plotted for three different combinations of the Rayleigh and Darcy numbers (open colored symbols). The theoretical scalings (solid black line) correspond to the large Schmidt solution (13) derived by Spalding and Cruddace [24] for a line source.

and $g(\eta)$ must be obtained numerically by integrating $(\mathrm{C} 8)$ with $g(0)=0, g^{\prime}(0)=g_{0}^{\prime}$, and $g^{\prime \prime}(0)=$ $-1 /\left(2 g_{0}^{\prime}\right)$. This solution for a line source is compared to the numerical profiles obtained for an extended source in Figs. 8(b) and 8(c). In agreement with the theory, the numerical data collapse fairly well when $c$ is renormalized by $z^{-3 / 5}$ and $w$ is renormalized by $z^{1 / 5}$ and that these quantities are plotted as a function of $x / z^{2 / 5}$. However, self-similarity is reached for a larger $z$ than in the previous Darcy regime: The data at $z=0.5$ (cyan symbols) do not collapse very well with the other values at larger $z$. These plots clearly confirm that the solute is mainly located near the axis of the plume whereas the velocity profile extends very far from the axis as was visible qualitatively on the bi-dimensional fields of Fig. 8. This can be understood intuitively since the concentration diffuses much less than the velocity at large Schmidt number and the ratio $\sigma_{w} / \sigma$ is expected to be on the order of $\sqrt{\mathrm{Sc}}$. Near the axis, the profile of concentration is close to the solution (13) of Spalding and Cruddace [24]. This is a good validation of the theory since the only parameter $\mathrm{Nu}$ is taken from the numerical simulations rather than adjusted for a best fit of the data. However, the concentration profile is slightly larger and thicker than The solution of Spalding and Cruddace [24] away from the axis (by about 30\%). The maximum vertical velocity component is also $30 \%$ smaller than the theoretical prediction.

The numerical axial $z$ profiles of the maximum concentration $c_{0}$, width of the concentration profile $\sigma$, maximum velocity $w_{0}$, and corresponding width $\sigma_{w}$, computed for three different combinations of Rayleigh and Darcy numbers, are displayed in Fig. 9. For the largest Darcy numbers, the scaling exponents seem to be in fair agreement with the predictions of the solution of Spalding and Cruddace [24] (indicated by solid lines). As is already visible in the bi-dimensional velocity field, it is interesting to note that the maximum velocity $w_{0}$ increases with $z$ whereas it is a decreasing function of $z$ in the Darcy regime [see Fig. 6(c)]. This explains why the $z$ profile of maximum velocity is flat in the experiments which correspond to flow conditions that are intermediate between the two regimes.

\section{Scaling laws-Transition between Darcy and Brinkman regimes}

In order to obtain a consistent picture of the plume's dynamics, that is, to say scaling laws which make the experimental and numerical data collapse on single generic curves, we perform in the following a scaling analysis of the problem for both the Darcy and the Brinkman regimes, based on mass conservation. The approach adopted to derive the scaling laws is depicted in Fig. 10. For each regime, the lateral flux $\phi_{l}$ of carbon dioxide in the region located underneath the meniscus (above the neck) is balanced by the vertical flux $\phi_{v}$ of carbon dioxide in the region where the velocity is roughly directed along the vertical axis (below the neck). On the other hand, the vertical pressure gradient is systematically neglected since the flow within the plume is essentially parallel and the gradient of the nondimensional $p$ outside the plume is zero (see more detailed arguments at the end of Sec. IIC), and the concentration is considered to be on the order of the saturation 


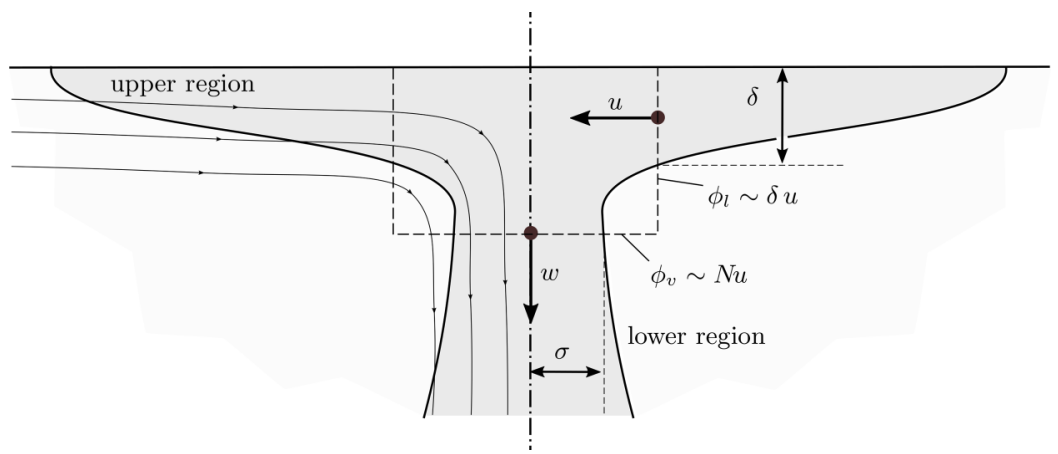

FIG. 10. Simplified picture of the plume suitable for the derivations of scaling laws of the vertical velocity and concentration width and Nusselt number with respect to the Rayleigh and Darcy numbers.

concentration-i.e., $c \sim 1$. Note also that the scaling laws for the Nusselt number will be given by the relationship $\phi_{v} \sim \phi_{l} \sim \mathrm{Nu}$.

A simple scaling law for the lateral flux as a function of the velocity can be derived by assuming that the $\mathrm{CO}_{2}$-enriched layer which builds up underneath the meniscus grows according to a simple diffusive law. Thus, one can write the order of magnitude of the lateral flux $\phi_{l}$ as the product $u \delta$, where $u$ denotes the lateral component of the velocity and $\delta \sim u^{-1 / 2}$ refers to the thickness of the diffusive layer when advected over a length equal to $1 / 2$. As $u$ and the vertical component $w$ in the lower region are roughly proportional to each other, one finally obtains $\phi_{l} \sim w^{1 / 2}$. In the steady state, this lateral flux is equal to the vertical flux in the plume, which is by definition equal to the Nusselt number. This leads to the equation,

$$
w \sim \mathrm{Nu}^{2}
$$

These crude scaling laws for the lateral and vertical fluxes are used below to derive approximate scaling laws for $\mathrm{Nu}, w, c$, and $\sigma$ with respect to the Rayleigh and Darcy numbers.

Darcy regime. Equating this scaling law (15) and the velocity given in Wooding's solution [23] (10) at $x=0$ and $z$ on the order of 1 leads to the following scaling laws in the Darcy regime:

$$
\mathrm{Nu} \sim \mathrm{Ra}^{1 / 2}, \quad w \sim \mathrm{Ra}, \quad \sigma \sim \mathrm{Ra}^{-1 / 2} .
$$

As expected, the Darcy number is not involved in these scalings since Da does not appear in the Darcy equation. Interestingly, introducing the above scaling law for the Nusselt number in Wooding's solution [23] (10) leads to $c \sim 1$ at $z=1$ and $x=0$.

Brinkman regime. Likewise, equating the scaling law (15) and the velocity given in the solution of Spalding and Cruddace [24] (13) at $x=0$ and $z$ on the order of 1 leads to the following scaling laws in the Brinkman regime:

$$
\mathrm{Nu} \sim\left(\frac{\mathrm{Ra}}{\mathrm{Da}}\right)^{1 / 4}, \quad w \sim\left(\frac{\mathrm{Ra}}{\mathrm{Da}}\right)^{1 / 2}, \quad \sigma \sim\left(\frac{\mathrm{Ra}}{\mathrm{Da}}\right)^{-1 / 4} .
$$

Again, the previous scaling laws indicate that the solution of Spalding and Cruddace [24] (13) only depends on the ratio Ra/Da since the Rayleigh and Darcy numbers only appear in the Brinkman equation through that ratio. It can be noted that introducing this scaling on the Nusselt number in the solution of Spalding and Cruddace [24] (13) implies that the concentration is on the order of 1 at $z=1$. 

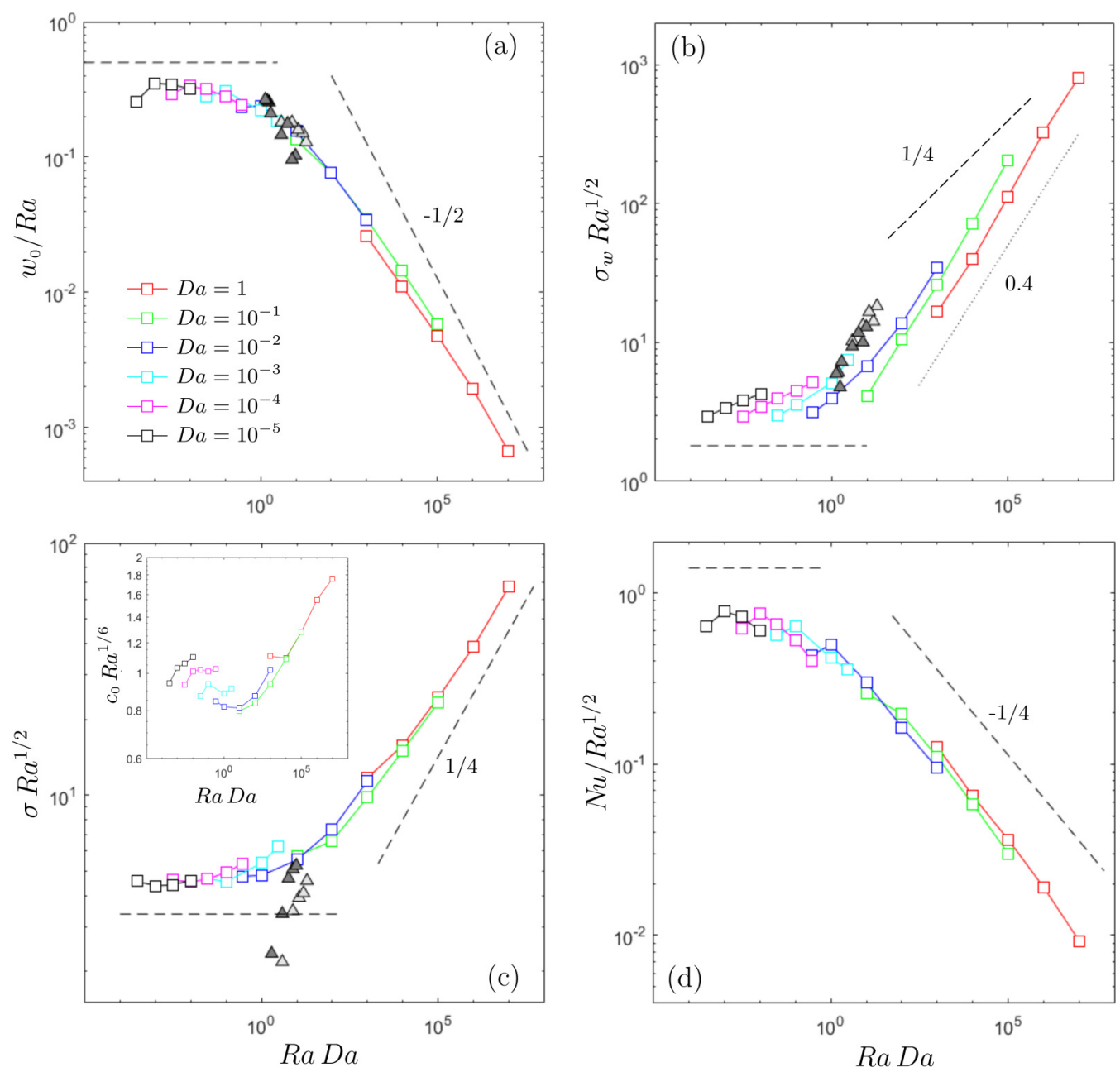

FIG. 11. Rescaled (a) maximum of the vertical velocity, (b) width of the velocity profile, (c) width of the concentration profile, and (d) Nusselt number, plotted with respect to the key parameter of the problem Ra Da . Experimental data are plotted using dark gray $\left(\mathrm{Da}=5.2 \times 10^{-4}\right)$ and light gray $\left(\mathrm{Da}=1.3 \times 10^{-3}\right)$ triangles. All the quantities are extracted/measured at $z=1$. The rescaled maximum concentration is plotted in the inset of (c) for information.

Based on the scaling laws derived above, one finally obtains the transition criterion,

$$
\mathrm{Ra} \mathrm{Da} \sim 1,
$$

which determines the crossover between low Darcy and large Darcy (Brinkman) regimes.

Thus, all quantities must be plotted with respect to the relevant dimensionless quantity $\mathrm{Ra} \mathrm{Da}$ as performed in Fig. 11. Scaling laws (16) and (17) can also be used as a guide to plot the quantities of interest $w_{0}, \sigma, \sigma_{w}$, and $\mathrm{Nu}$ in a unified way so that they collapse on single curves over the entire range of explored dimensionless numbers. To do so, the characteristics are divided by their scaling laws in the Darcy regime in order to get constant values at $\mathrm{Ra} \mathrm{Da} \ll 1$. A plateau is indeed observed for the ratio of the vertical velocity component to the Rayleigh number, which is plotted in Fig. 11(a). Furthermore, the numerical data also collapse on a single curve in the Brinkman regime. 
This can be understood from the scaling laws (17) which imply that $w_{0} / \mathrm{Ra} \sim(\mathrm{Ra} \mathrm{Da})^{-1 / 2}$ as indeed observed numerically.

The same tendencies can be observed for the width of the plume $\sigma$ and the Nusselt number which are in excellent agreement with the scalings laws. Empirical laws for the Nusselt number can, thus, be given in the Darcy regime $(\mathrm{Ra} \mathrm{Da} \ll 1)$ as

$$
\mathrm{Nu}=0.7 \mathrm{Ra}^{1 / 2}
$$

and in the Brinkman regime,

$$
\mathrm{Nu}=0.6\left(\frac{\mathrm{Ra}}{\mathrm{Da}}\right)^{1 / 4},
$$

with an error of $15 \%$ on the constants. Introducing these laws in the corresponding line source solutions gives the complete concentration and velocity fields in the entire domain.

It can be noted that the width of the velocity profile $\sigma_{w}$ decreases slightly more slowly than the predicted scalings. It scales as $(\mathrm{RaDa})^{-0.38}$ in the Darcy regime rather than $(\mathrm{Ra} \mathrm{Da})^{-0.5}$ and as $(\mathrm{Ra} \mathrm{Da})^{-0.1}$ rather than $(\mathrm{RaDa})^{-0.25}$ in the Brinkman regime. This could be due to the slight dependence of the concentration as $\mathrm{Ra}^{-1 / 6}$ which can be observed in the subset of Fig. 11(c). In the scalings laws derived above, it was indeed assumed that the concentration is independent of the Rayleigh and Darcy numbers. It is not clear whether this effect is due to the fact that the simulations are performed in an intermediate regime or if it is due to the fact that the measurements are performed too close to the extended source. However, this effect is fairly small since the concentration only varies by a factor of 5 when Ra Da varies over 12 orders of magnitude.

\section{Comparison between experimental and numerical results}

The experimental values obtained by PIV are also plotted in Figs. 11(a) and 11(b) as gray symbols. The velocities are in excellent agreement with the numerical results. It can be noted that they are in the intermediate regime between the Darcy and the Brinkman regimes. The width of the velocity profiles $\sigma_{w}$ which are obtained experimentally at $\mathrm{Da}=10^{-3}$ are also in excellent agreement with the numerical simulations (which correspond to the cyan symbols). Finally, the width of the concentration profiles are plotted in Fig. 11(c) for information although there is a large uncertainty in the measurement technique as discussed in Sec. II B. The width inferred from the $p \mathrm{H}$ measurements is on the same order of magnitude as that obtained in the numerical simulations. However, whereas numerical simulations and experiments match each other reasonably well for the largest Rayleigh numbers, the agreement deteriorates for lower Rayleigh numbers where experimental values are, at least, twice as small as numerical ones.

\section{CONCLUSION}

The dynamics of a bi-dimensional plume has been studied experimentally in a Hele-Shaw cell and numerically using two-dimensional (2D) Darcy-Brinkman equations. The plume was generated by dissolution of $\mathrm{CO}_{2}$ at the free surface over a length $L_{s}$, which allows for the definition of a length-based Rayleigh number. In contrast to plumes generated by a line source, the Nusselt number is unknown and has been determined empirically as a function of the Rayleigh number. Two regimes have been observed for which the scalings have been predicted theoretically. For $\mathrm{Ra} D \mathrm{Da} \ll 1$, the plume is in the Darcy regime, and the Nusselt number is given by $\mathrm{Nu}=0.7 \mathrm{Ra}^{1 / 2}$, independently of the Darcy number. For Ra Da $\gg 1$, the Brinkman term in the flow equation becomes larger than the Darcy term, and the Nusselt number is given by $\mathrm{Nu}=0.6(\mathrm{Ra} / \mathrm{Da})^{1 / 4}$. In both regimes, the velocity and concentration fields are very close to the line source solutions, given by Wooding [23] for the Darcy regime and by Spalding and Cruddace [24] for the Brinkman regime.

As a building block of convection, plumes have been largely studied in the case of point or line sources. However, there have been very few studies extending these models to the cases of 
extended sources. The empirical laws presented in this paper will be useful to model boundary layers in convection. However, such models will probably require a prediction of the number of plumes, which deserves a study in itself. This could be maybe studied by looking at the interaction of several plumes.

Another topic of interest is the stability of these plumes. Indeed, it has been observed numerically and experimentally that the plume could destabilize at large Rayleigh numbers, giving rise to periodic puffs. Predicting the frequency and the onset of this instability would be extremely interesting for the study of turbulent convective boundary layers.

Finally, porous media flows described on the continuum scale are known to give rise to hydrodynamics dispersion of the advected scalar, a property which must be taken into account, e.g., when modeling the convective dissolution of $\mathrm{CO}_{2}$ in deep geological formation [29]. It is not clear how the models presented in this paper would change in the presence of hydrodynamic dispersion. This could also be studied in a future work.

\section{APPENDIX A: CONCENTRATION PROFILES MEASURED FROM $p H-S E N S I T I V E$ DYE VISUALIZATION}

The $p \mathrm{H}$-sensitive dye visualizations have been used to extract the characteristics of the plume. From the concentration map $C(x, z)$, the transverse profiles were extracted as shown in Fig. 12 for $z=L_{s}$. The profile is very close to the profile derived by Wooding [23], see Eq. (10). The width $\sigma$ is plotted in Fig. 12(c) as a function of $z$ for $E=1 \mathrm{~mm}$ and $P_{\mathrm{CO}_{2}}=1$ bar. The scaling exponent is in good agreement with the solution of Spalding and Cruddace [24], which predicts an exponent of $2 / 3$. The width has been measured for $P_{\mathrm{CO}_{2}}=1$ to 5 bars and for $E=0.5$ and $1 \mathrm{~mm}$ and its dependence on $\mathrm{Ra}$ and $\mathrm{Da}$ is reported in Fig. 11(c). They are smaller than the numerical results by a factor up to 2. This large error is inherent to this measurement method. Indeed, the maximum concentration was also extracted from the profiles. They were found in some cases to be up to ten times larger or smaller than the known concentration at the surface. These errors are due to small variations of intensity in the images, which lead to large variations of the concentration. For example, a small increase of $10 \%$ in the intensity (due to variation of the illumination, change of temperature, etc.) creates a fluctuation of 0.2 in the $p \mathrm{H}$, which leads to a concentration 2.5 times larger than its correct value using the formula $p \mathrm{H}=\left(p C+p K_{a}\right) / 2$. Surprisingly, the uncertainties on the width were much smaller because the large-scale fluctuations of intensity do not bias the value of $\sigma$. Indeed, it corresponds to the second derivative of $p C(x)$, which is not altered by the addition of a uniform fluctuation. This is why the width is studied in detail in the core of the article whereas the maximum concentration is not.
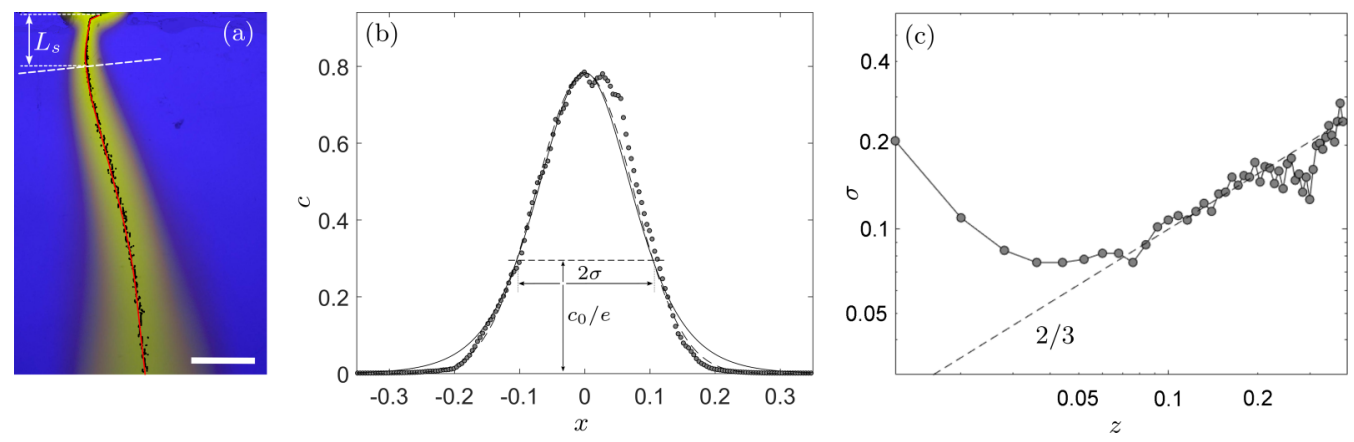

FIG. 12. (a) Concentration map of the plume for $E=1 \mathrm{~mm}, P_{\mathrm{CO}_{2}}=1 \mathrm{bar}$. The length of the white scale bar is $4 \mathrm{~mm}$. (b) Corresponding transverse profile at $z=L_{s}$. The dashed line corresponds to a Gaussian fit $\propto$ $\exp \left(-x^{2} / \sigma^{2}\right)$ whereas the solid line corresponds to a fit $\propto[\cosh (1.085 x / \sigma)]^{-2}$. (c) Width $\sigma$ of the concentration profile as a function of the altitude. 


\section{APPENDIX B: SELF-SIMILAR PLUME GENERATED BY A LINE SOURCE \\ IN THE DARCY REGIME}

The goal of this Appendix is to derive the solution to the coupled flow and transport problem for a line source of concentration with a flux $\mathrm{Nu}$ defined by

$$
\mathrm{Nu}=\int_{-\infty}^{+\infty} c w d x
$$

The incompressible $2 \mathrm{D}$ velocity field can be searched as

$$
u=-\frac{\partial \Psi}{\partial z}, \quad w=\frac{\partial \Psi}{\partial x},
$$

where the stream function $\Psi$ depends algebraically on $z$. From dimensional analysis, it can be shown that the stream function must be of the form

$$
\Psi(x, z)=\mathrm{Nu}^{1 / 3} \mathrm{Ra}^{1 / 3} z^{1 / 3} f(\eta) \quad \text { with } \quad \eta=\frac{\mathrm{Nu}^{1 / 3} \mathrm{Ra}^{1 / 3}}{z^{2 / 3}} x .
$$

For large Schmidt numbers, the term on the left-hand side of (7) can be neglected. In the Darcy regime, the Brinkman term can be neglected as well so that the Navier-Stokes equation simplifies to

$$
\nabla p=-\boldsymbol{u}+\operatorname{Ra} c z
$$

For thin plumes, the pressure at the center of the plume is equal to the pressure outside the plume as discussed previously. The vertical pressure gradient can, thus, be neglected. The vertical component of (B4), thus, becomes

$$
c=\frac{w}{\mathrm{Ra}}
$$

which can be calculated using (B3) and (B2),

$$
c=\frac{\mathrm{Nu}^{2 / 3}}{z^{1 / 3} \mathrm{Ra}^{1 / 3}} f^{\prime}(\eta) .
$$

Introducing this expression in the steady version of Eq. (8) and using the expression for $u$ and $w$ given by the stream function leads to a simple ordinary differential equation,

$$
3 f^{\prime \prime \prime}+f f^{\prime \prime}+f^{\prime 2}=0 .
$$

The general odd solution of this equation is

$$
f(\eta)=A \tanh (A \eta / 6)
$$

The constant $A$ is found by stating that the flux must be equal to $\mathrm{Nu}$, leading to $A=(9 / 2)^{1 / 3}$. The self-similar solutions for the vertical velocity component and concentration are, thus, given by

$$
w=\operatorname{Ra} c=\frac{2}{3}\left(\frac{9 \mathrm{Nu} \mathrm{Ra}}{16}\right)^{2 / 3} z^{-1 / 3} \cosh ^{-2}\left[\frac{1}{3}\left(\frac{9 \mathrm{Nu} \mathrm{Ra}}{16}\right)^{1 / 3} \frac{x}{z^{2 / 3}}\right] .
$$

This solution was given by Wooding [23] who noted that it was identical to the solution of a momentum jet given by Schlichting and Kestin [30]. 


\section{APPENDIX C: SELF-SIMILAR PLUME GENERATED BY A LINE SOURCE IN THE BRINKMAN REGIME}

At large Darcy numbers the steady versions of the depth-averaged Navier-Stokes Eq. (7) and diffusion-advection Eq. (8) become

$$
\begin{gathered}
\left(\boldsymbol{u} \cdot \nabla_{\|}\right) \boldsymbol{u}=\frac{5}{6} \operatorname{Sc}\left[-\nabla_{\|}^{2} u+\left(\frac{\mathrm{Ra}}{\mathrm{Da}}\right) c z\right], \\
\left(\boldsymbol{u} \cdot \nabla_{\|}\right) c=\nabla_{\|}^{2} c .
\end{gathered}
$$

In Eq. (C1), the pressure gradient term has been neglected for the same reason as in the Darcy equation (B4). From dimensional analysis, it can be shown that the concentration and the vertical component of the stream function $\Psi$ defined by $u=-\partial_{z} \Psi$ and $w=\partial_{x} \Psi$ can be written

$$
c=\frac{5}{6} \operatorname{Sc}\left(\frac{\mathrm{Da}}{\mathrm{Ra}}\right) \Lambda^{4 / 5} z^{-3 / 5} \theta(\eta) \quad \text { and } \quad \Psi=\frac{5}{6} \operatorname{Sc} \Lambda^{1 / 5} z^{3 / 5} g(\eta),
$$

with

$$
\Lambda=\frac{36}{25} \frac{\mathrm{NuRa}}{\mathrm{DaSc}^{2}} \quad \text { and } \quad \eta=\frac{\Lambda^{1 / 5}}{z^{2 / 5}} x
$$

Introducing the forms (C3) in Eq. (C2) leads, after some algebra, to

$$
\theta^{\prime \prime}+\frac{1}{2} \operatorname{Sc}(g \theta)^{\prime}=0
$$

where the primes denote the successive derivative with respect to $\eta$. For large Schmidt numbers, the vertical velocity can be considered as constant in the vicinity of the axis (within the concentration plume) so that the approximate form $g(\eta) \simeq g_{0}^{\prime} \eta$ can be introduced in Eq. (C5), leading to the linear differential equation,

$$
\theta^{\prime \prime}+\frac{1}{2} \operatorname{Scg}_{0}^{\prime}(\eta \theta)^{\prime}=0
$$

The previous equation can be straightforwardly solved to obtain the function $\theta$ in the form

$$
\theta(\eta)=\left(\frac{\mathrm{Sc}}{4 \pi g_{0}^{\prime}}\right)^{1 / 2} \exp \left(-\frac{\mathrm{Sc} g_{0}^{\prime}}{4} \eta^{2}\right)
$$

where the constant $g_{0}^{\prime}$ is yet to be determined.

Now, introducing the forms (C3) in the $z$ component of Eq. (C1) yields

$$
g^{\prime \prime \prime}+\frac{3}{5} g g^{\prime \prime}-\frac{1}{5} g^{2}-\theta=0 .
$$

Equation (C8) is solved numerically with initial conditions $g(0)=0, g^{\prime}(0)=g_{0}^{\prime}$, and a third initial condition which can be found by integrating theoretically (C8) from $\eta=0$ to $\varepsilon$ with $\mathrm{Sc}^{-1 / 2} \ll \varepsilon \ll$ 1 , which leads to $g^{\prime \prime}(0)=\left(2 g_{0}^{\prime}\right)^{-1}$. This numerical solution diverges at large $\eta$ except if $g_{0}^{\prime}=0.9335$ as found by Spalding and Cruddace [24].

[1] S. Bachu, Screening and ranking of sedimentary basins for sequestration of $\mathrm{CO}_{2}$ in geological media in response to climate change, Environ. Geol. 44, 277 (2003).

[2] IPCC - Report on Carbon Dioxide Capture and Storage, edited by B. Metz, O. Davidson, H. de Coninck, M. Loos, and L. Meyer (Cambridge University Press, UK, 2005).

[3] Northern Light CCS website, https://northernlightsccs.com/en/about, 2021.

[4] A.-K. Furre, O. Eiken, H. Alnes, J. N. Vevatne, and A. F. Kiær, 20 years of monitoring $\mathrm{CO}_{2}$-injection at Sleipner, Energy Procedia 114, 3916 (2017).

[5] H. Huppert and J. Neufeld, The fluid mechanics of carbon dioxide sequestration, Annu. Rev. Fluid Mech. 46, 255 (2014). 
[6] T. J. Kneafsey and K. Pruess, Laboratory experiments and numerical simulation studies of convectively enhanced carbon dioxide dissolution, Energy Procedia 4, 5114 (2011).

[7] A. Slim, M. Brandi, J. Miller, and L. Mahadevan, Dissolution-driven convection in a Hele-shaw cell, Phys. Fluids 25, 024101 (2013).

[8] R. Outeda, C. El Hasi, A. D’Onofrio, and A. Zalts, Experimental study of linear and nonlinear regimes of density-driven instabilities induced by $\mathrm{CO}_{2}$ dissolution in water, Chaos 24, 013135 (2014).

[9] A. Vreme, F. Nadal, B. Pouligny, P. Jeandet, G. Liger-Belair, and P. Meunier, Gravitational instability due to the dissolution of carbon dioxide in a Hele-Shaw cell, Phys. Rev. Fluids 1, 064301 (2016).

[10] V. Loodts, B. Knaepen, L. Rongy, and A. De Wit, Enhanced steady-state dissolution flux in reactive convective dissolution, Phys. Chem. Chem. Phys. 19, 18565 (2017).

[11] J. Ennis-King, I. Preston, and L. Paterson, Onset of convection in anisotropic porous media subject to a rapid change in boundary conditions, Phys. Fluids 17, 084107 (2005).

[12] A. Riaz, M. Hesse, H. A. Tchelepi, and F. M. Orr, Onset of convection in a gravitationally unstable diffusive boundary layer in porous media, J. Fluid Mech. 548, 87 (2006).

[13] M. T. Elenius and K. Johannsen, On the time scales of nonlinear instability in miscible displacement porous media flow, Comput. Geosci. 16, 901 (2012).

[14] N. Tilton and A. Riaz, Nonlinear stability of gravitationally unstable, transient, diffusive boundary layers in porous media, J. Fluid Mech. 745, 251 (2014).

[15] C. S. Yih, Free convection due to a point source of heat, in Proceedings of the First U.S. National Congress of Applied Mechanics (ASME, New York, 1951), pp. 941-947.

[16] T. Fujii, Theory of the steady laminar natural convection above a horizontal line heat source and a point heat source, Int. J. Heat Mass Transf. 6, 597 (1963).

[17] L. Pera and G. Gebhart, On the stability of laminar plumes : Some numerical solutions and experiments, Int. J. Heat Mass Transfer 14, 975 (1971).

[18] M. G. Worster, The axisymmetric laminar plume: Asymptotic solution for large Prandtl number, Stud. Appl. Math. 75, 139 (1986).

[19] R. J. Whittaker and J. R. Lister, Steady axisymmetric creeping plumes above a planar boundary. Part 1. A point source, J. Fluid Mech. 567, 361 (2006).

[20] A. Umemura and F. H. Busse, Axisymmetric convection at large Rayleigh and infinite Prandtl number, J. Fluid Mech. 208, 459 (1989).

[21] R. J. Whittaker and J. R. Lister, Steady axisymmetric creeping plumes above a planar boundary. Part 2. A distributed source, J. Fluid Mech. 567, 379 (2006).

[22] F. Nadal, P. Meunier, B. Pouligny, and E. Laurichesse, Stationary plume induced by carbon dioxide dissolution, J. Fluid Mech. 719, 203 (2013).

[23] R. A. Wooding, Convection in a saturated porous medium at large Rayleigh number or Péclet number, J. Fluid Mech. 15, 527 (1963).

[24] D. B. Spalding and R. G. Cruddace, Theory of the steady laminar buoyant flow above a line heat source in a fluid of large Prandtl number and temperature-dependent viscosity, Int. J. Heat Mass Transfer. 3, 55 (1961).

[25] P. Meunier and T. Leweke, Analysis and optimization of the error caused by high velocity gradients in particle image velocimetry, Exp. Fluids 35, 408 (2003).

[26] T. Lacassagne, S. Simoens, M. El Hajem, and J.-Y. Champagne, Ratiometric, single-dye, pH-sensitive inhibited laser-induced fluorescence for the characterization of mixing and mass transfer, Exp. Fluids 59, 21 (2018).

[27] A. Hebach, A. Oberhof, and N. Dhamen, Density of water + carbon dioxyde at elevated pressures: Measurements and correlation, J. Chem. Eng. Data 49, 950 (2004).

[28] E. Åkervik, L. Brandt, D. S Henningson, J. Hœpffner, O. Marxen, and P. Schlatter, Steady solutions of the navier-stokes equations by selective frequency damping, Phys. Fluids 18, 068102 (2006).

[29] J. J. Hidalgo and J. Carrera, Effect of dispersion on the onset of convection during $\mathrm{CO}_{2}$ sequestration, J. Fluid. Mech. 640, 441 (2009).

[30] H. Schlichting and J. Kestin, Boundary Layer Theory, 9th ed. (Springer-Verlag, Berlin, Heidelberg, 2017). 